\title{
IAD Method Applied to Reactor Physics Problems
}

\author{
Phan Huy Thien ${ }^{1}$ \\ ${ }^{1}$ Department of Theoretical Physics, Faculty of Physics, Hanoi University of Science, \\ Vietnam National University \\ 334 Nguyen Trai Street, Thanh Xuan, Hanoi, Vietnam
}

\begin{abstract}
The neutron diffusion equations depen on the space and times with multi-group of delayed neutrons are a couple of the stiff nonlinear partial differential equations. Application of finite difference method for simplified equations to partial differential equations often then is rewritten as a matrix form. The general solution of the matrix differential equation contains the exponential function of the coefficient matrix. The numerical techniques for processing the exponential function of the coefficient matrix are presented using an alytical method and fundamental matrix method. Method IAD (average method integral derivative) to define the boundary conditions for the specific geometry of the reactor. Eigenvalues matrix coefficients are calculated by MATLAB program. Numerical results are compared with the other results.
\end{abstract}

Keywords: The Neutron field in Reactor, Boltzman transport equation, boundary conditions for the specific geometry of the reactor

\section{Introduction}

Neutron diffusion equation describing the density of neutrons in a nuclear reactor core. Many methods have been proposed to calculate the neutron diffusion equation is mostly based on the finite difference method (Finite difference method FDM) applied to the domain geometry as in the case of twodimensional XY or RZ. With complex geometries, using the finite element method (Finite element method - FEM). In addition, the analytical method [1], [2], Monte Carlo method [7]. The Boltzmann equation in nuclear reactors have been developed with the program complex calculations. However, there are many difficulties in the calculation depends on the application of the method to each specific problem, they relate to the timing and geometry of the system to be calculated. Some of authors using mixed methods approach between methods to take advantage of these methods simultaneously overcoming the disadvantages and shortcomings of each method.

With the problem of crack boundary or not enough smoothness, with the derivative in the classical sense of the functions involved in boundary conditions, finding ways to overcome or slippery spots or singular about the very important. The concept of the average integral derivative (AD method) allows in many cases find a way to show a consistent approach to the formal point and the singularity but the average derivative in the sense of achievement feces. Some of the results using the concept of generalized derivative in the sense that the average integral given in [13] method called $\mathrm{AD}$. AD method applied to solve the nuclear reactors proposed by us, in combination with traditional methods can solve the equation as a consistent algorithm including singularities where it is not with normal derivative.

The AD method combine with some methods such as multistep method, the method rather bizarre integral finite sum was some authors to use and has good results in many problems - especially theoretical fault (crack theory) of mechanics of continuous environments. Author J.J. Golecki has focused on the application method "Phan Van Hap" in a series of articles published in scientific journals from 1979 to
2007 [11].

In the last six decades, the calculation and design of nuclear reactors were significant steps, along with the development of computers. We build neutron transport equation, and consider the method of calculation, the successes and limitations of previous methods, the proposed method is the average integral derivative (AD method) to the solution neutron transport equations to describe the physical processes taking place in a nuclear reactor core. Results after solving the problem of nuclear reactor neutron flux is given for each group of energy and space coordinates and effective multiplication factor $k_{\text {eff }}$.

\section{The Time Dependent Neutron Diffusion Problem}

The multi-energy group nuclear reactor kinetics equations with multi-group delayed neutrons can be written in the following form [5]:

$$
\begin{aligned}
& \frac{1}{v_{g}} \frac{\mathbb{q}}{\mathbb{\top} t} \mathrm{~F}_{g}(\mathbf{r}, t) \\
& =\tilde{\mathrm{N}} \times D_{g}(\mathbf{r}) \tilde{\mathrm{N}} \mathrm{F}_{g}(\mathbf{r}, t)-\mathrm{S}_{a_{g}} \mathrm{~F}_{g}(\mathbf{r}, t)-\stackrel{\mathrm{a}}{g \bowtie g}_{\mathrm{O}^{G}}^{\mathrm{S}} \mathrm{S}_{s_{g, g \phi}} \mathrm{F}_{g}(\mathbf{r}, t)
\end{aligned}
$$

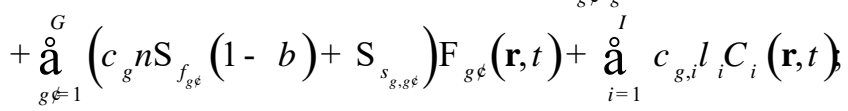

$$
\begin{aligned}
& g=1,2, \ldots, G \\
& \frac{\mathbb{q}}{\mathbb{\Phi} t} C_{i}(\mathbf{r}, t)=b_{i} \stackrel{\stackrel{a}{\mathrm{a}}}{g} n \mathrm{~S}_{f_{g}} \mathrm{~F}_{g}(\mathbf{r}, t)-l_{i} C_{i}(\mathbf{r}, t)
\end{aligned}
$$

where $\mathbf{r}$ is the position vector $(\mathrm{cm}), t$ is the time $(s)$, $\mathrm{F}_{g}(\mathbf{r}, t)$ is the scalar neutron flux $\left(\mathrm{cm}^{-2} \mathrm{~s}^{-1}\right)$ in group $g$, $C_{i}(\mathbf{r}, t)$ is the concentration of delayed neutron precursors $\left(\mathrm{cm}^{3}\right)$ in group $i, v_{g}$ is the mean velocity of the neutron $\left(\mathrm{cm} \mathrm{s}^{-1}\right)$ in group $g, D_{g}(\mathbf{r})$ is the diffusion 


\section{International Journal of Science and Research (IJSR) \\ ISSN (Online): 2319-7064 \\ Index Copernicus Value (2013): 6.14 | Impact Factor (2014): 5.611}

coeffcient $(\mathrm{cm})$ in group $g, \mathrm{~S}_{a_{g}}(\mathbf{r}, t)$ is the absorption cross-section $\left(\mathrm{cm}^{-1}\right)$ in group $g, \mathrm{~S}_{f_{g}}(\mathbf{r})$ is the fission cross-section $\left(\mathrm{cm}^{-1}\right)$ in group $\mathrm{g}, \mathrm{S}_{s_{g, g \xi}}(\mathbf{r})$ is the scattering cross-section $\left(\mathrm{cm}^{-1}\right)$ from group $g \notin$ to group $g$ such that $\mathrm{S}_{s_{g, g \phi}}(\mathbf{r})=0$ for $g \notin>g, n$ is the mean number of fission neutrons, $c_{g}$ is the spectrum of prompt neutrons in group $g$, $c_{g, i}$ is the spectrum of $i$-group delayed neutrons in group $g$, $l_{i}$ is the decay constant $\left(s^{-1}\right)$ of group $i$ precursors, $b_{i}$ is the fraction of delayed neutrons in group $i$, and $b=\stackrel{I}{a} \stackrel{a}{i=1}_{i} b_{i}$ is the total fraction of delayed neutrons.

Using the finite difference method, the equations (1) and (2) lead to the following matrix form

$$
\frac{d \mathrm{Y}(t)}{d t}=\mathrm{AY}(t)+\mathbf{B}
$$

where,

$$
\mathrm{Y}(t)=\begin{array}{llllllll}
\text { 委 }_{1} & \overline{\mathrm{F}}_{2} & \ldots & \overline{\mathrm{F}}_{G} & C_{1} & C_{2} & \ldots & C_{I} \stackrel{\mathrm{u}}{\mathrm{u}}
\end{array}
$$

Here, $\overline{\mathrm{F}}_{1}, \overline{\mathrm{F}}_{2}, \ldots, \overline{\mathrm{F}}_{G}$ Here is the neutron flux in equation (1) and (2) has been averaging by method $\mathrm{AD}$ in the shape boundary conditions given [14]

$$
\lim _{d \mathbb{B} 0} \frac{1}{2 d} \mathrm{O}_{-d}{ }^{d} \frac{\mathrm{F}_{i}\left(x_{0}+t h\right)-\mathrm{F}_{i}\left(x_{0}\right)}{t} d t=\overline{\mathrm{F}}_{i}\left(x_{0}, h\right)^{\mathrm{o}} \overline{\mathrm{F}}_{i}
$$

The boundary conditions for the g-group neutron fluxes and initial conditions

$$
\begin{aligned}
& \left\lfloor\sigma_{g} \Phi_{g}+\tau_{g} \frac{\partial \Phi_{g}}{\partial n}\right\rfloor_{\partial V}=0 ; g=1, \ldots, G ; \\
& \Phi_{g}(\mathbf{r}, 0)=\Phi_{g}(\mathbf{r}) \\
& C_{i}(\mathbf{r}, 0)=C_{i}(\mathbf{r}) ; i=1, \ldots, I
\end{aligned}
$$

Where $\partial / \partial u$ denotes the outward normal derivative at the point $\mathbf{r}_{\partial V}$ of reactor boundary $\partial V$.

Assume that, the matrices $\mathbf{A}$ và $\mathbf{B}$ are constant during the interval time $t_{m}$ and $t_{m+1}=t_{m}+h$ considering the length of the time interval is small. Then, the general analytical solution of equation (2) takes the following form:

$$
\mathrm{Y}\left(t_{m+1}\right)=\exp (h \mathbf{A}) \mathrm{Y}\left(t_{m}\right)+(\exp (h \mathbf{A})-\mathbf{I}) \mathbf{A}^{-1} \mathbf{B}(6)
$$

Using analytical methods to rewrite the exponential of the coefficient matrix analytic methods to develop a chain function

$$
\exp (h \mathbf{A})=\stackrel{̊}{\mathrm{a}}_{k=0}^{K} a_{k}(h \mathbf{A})^{k}
$$

in which $a_{0}, a_{2}, \ldots, a_{K}$ are the coefficients and $K=G+I-1$ is the number of rows and columns of the coefficient matrix A.
Coefficients $a_{k}$ can be determined by solving the following equation:

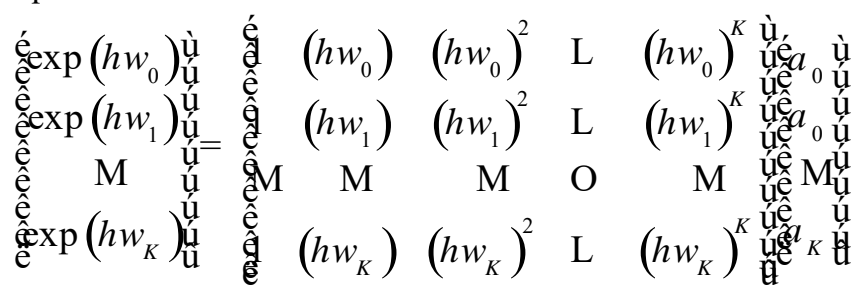

(8)

where $w_{0}, w_{1}, \ldots, w_{K}$ are the eigenvalues of the matrix $\mathrm{A}$. The eigenvalues are calculated numerically using MATLAB computer code based on Laguere's method.

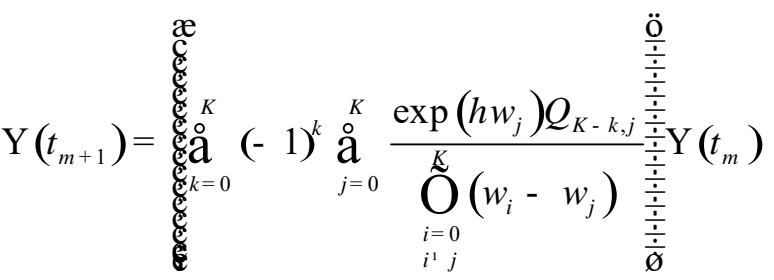

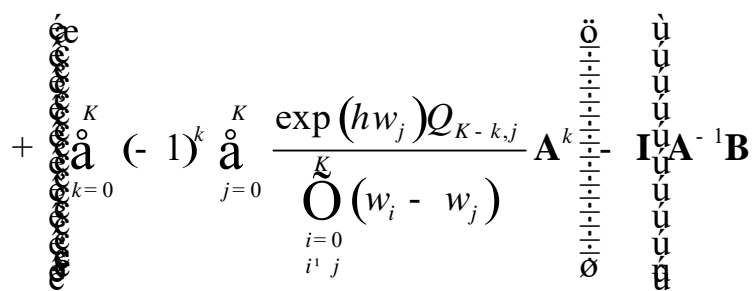

This equation represents the numerical technique for solving the space-time neutron diffusion equations with multi-group of delayed neutrons which based on analytical method for the exponential function of matrix $\mathbf{A}$, with IAD method.

The fundamental matrix and its inverse are calculated analytically for two energy groups and average one group of delayed neutron.

To study the influence of the delay neutrons and the effects of the source dominance with increasing subcriticaly a simple 1-dimensional homogeneous reactor with a source in the center region chosen and this case was studied in one-speed diffusion theory.

The neutron flux in one speed diffusion theory (with I precursor groups) is defined by

$$
\begin{aligned}
& \frac{1}{V} \frac{\partial \Phi}{\partial t}=D \frac{\partial^{2} \Phi}{\partial x^{2}}-\Sigma_{a} \Phi+(1-\beta) v \Sigma_{f} \Phi+\sum_{i=1}^{I} \lambda_{i} C_{i}+Q \\
& \frac{\partial C_{i}}{\partial t}=-\lambda_{i} C_{i}+\beta_{i} v \Sigma_{f} \Phi ; \quad i=1,2, \ldots, I .
\end{aligned}
$$

When the extrapolated length is neglected the boundary conditions are $\Phi\left(-\frac{a}{2}, t\right)=0$ and $\Phi\left(\frac{a}{2}, t\right)=0$, where a is the thickness of the slab reactor. When the source is turned on it is described as

$$
q(x)=\left\{\begin{array}{lc}
1 & -2 \leq x \leq 2 \\
0 & |x| \geq 2
\end{array}\right.
$$


International Journal of Science and Research (IJSR)

ISSN (Online): 2319-7064

Index Copernicus Value (2013): 6.14 | Impact Factor (2014): 5.611

The effective multiplication factor for such a system is

$$
k_{e f f}=\frac{v \Sigma_{f}}{\Sigma_{a}\left(1+L^{2}+B_{1}^{2}\right)}
$$

where the diffusion length $L=\sqrt{\frac{D}{\Sigma}}$ and $B_{1}=\left(\frac{\pi}{a}\right)$.

If the system is in a steady state $\left(\frac{\partial \Phi}{\partial t}=0, \frac{\partial C_{i}}{\partial t}=0\right)$ equation (10) reduces to

$D \frac{\partial^{2} \Phi}{\partial x^{2}}=\left(\Sigma_{a}-v \Sigma_{f}\right) \Phi-q ; C_{i}=\frac{\beta_{i} v \Sigma_{f}}{\lambda_{i}} \Phi ; i=1,2, \ldots, I$

The space dependence of the steady-state diffusion equation is discretized by finite differences. The $\mathrm{x}$-domain is divided into K sub-intervals with length $h$, which leads to

$$
\frac{\Phi\left(x_{k-1}\right)-2 \Phi\left(x_{k}\right)+\Phi\left(x_{k+1}\right)}{h^{2}}=\frac{\Sigma_{a}-v \Sigma_{f}}{D} \Phi\left(x_{k}\right)-\frac{q\left(x_{k}\right)}{D}
$$

Since the extrapolated distance is neglected the boundary conditions are given by $\Phi\left(x_{1}\right)=\Phi\left(x_{k}\right)=0$. This can be written in matrix vector notation with 3 main diagonal by writing

$$
\mathbf{A}=\left[\begin{array}{cccccc}
1 & 0 & 0 & 0 & 0 & 0 \\
-1 & 2+\frac{\Sigma_{a}-v \Sigma_{f}}{D} h^{2} & -1 & 0 & 0 & 0 \\
0 & -1 & 2+\frac{\Sigma_{a}-v \Sigma_{f}}{D} h^{2} & -1 & 0 & 0 \\
0 & 0 & -1 & \ddots & \ddots & \vdots \\
0 & 0 & 0 & \ddots & 2+\frac{\Sigma_{a}-v \Sigma_{f}}{D} h^{2} & -1 \\
0 & 0 & 0 & \ldots & 0 & 1
\end{array}\right]
$$

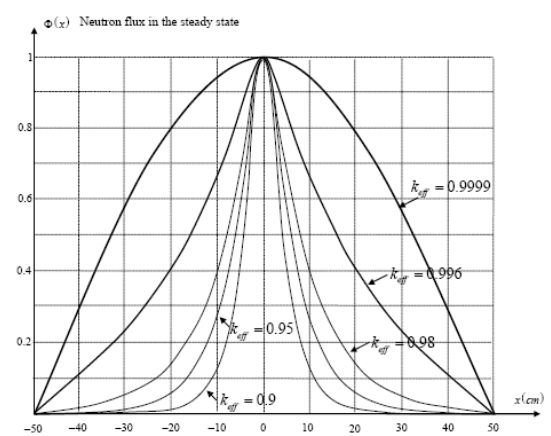

Figure 1: The neutron fluxes of the 1D diffusion problem for different $k_{\text {eff }}$.

With

$$
\vec{\Phi}=\left[\begin{array}{c}
\Phi\left(x_{1}\right) \\
\Phi\left(x_{2}\right) \\
\vdots \\
\Phi\left(x_{K}\right)
\end{array} \mid ; \quad \vec{q}=\frac{h^{2}}{D}\left\lfloor\begin{array}{c}
q\left(x_{1}\right) \\
q\left(x_{2}\right) \\
\vdots \\
q\left(x_{K}\right)
\end{array}\right\rfloor\right.
$$

Now use MATLAB can solve the following system

$$
\mathbf{A} \vec{\Phi}=\vec{q}
$$

Once the flux $\vec{\Phi}$ is known the steady state precursor concentrations $C_{i}$ can easily be calculated.

Calculations of the steady state neutron flux were performed for $k_{\text {eff }}$ ranging from 0.9 to 0.9999 . $k_{\text {eff }}$ was varied by choosing the appropriate fission cross section $\Sigma_{f}$. The steady state neutron flux is normalized and plotted in Fig. 1 for the different values of $k_{\text {eff }}$.

The two energy groups neutron diffusion equations governing the dynamic groups diffusion neutron flux and average one group of the precursor concentration of delayed neutrons behavior are written as follows

$$
\begin{aligned}
& \frac{1}{v_{1}} \frac{\partial}{\partial t} \Phi_{1}(\mathbf{r}, t)=D_{1} \nabla^{2} \Phi_{1}(\mathbf{r}, t)-\left(\Sigma_{a_{1}}+\Sigma_{s_{12}}\right) \Phi_{1}(\mathbf{r}, t) \\
& +v \Sigma_{f_{1}}(1-\beta) \Phi_{1}(\mathbf{r}, t)+v \Sigma_{f_{2}}(1-\beta) \Phi_{2}(\mathbf{r}, t)+\lambda C(\mathbf{r}, t), \\
& \frac{1}{v_{2}} \frac{\partial}{\partial t} \Phi_{2}(\mathbf{r}, t)=D_{2} \nabla^{2} \Phi_{2}(\mathbf{r}, t)-\Sigma_{a_{2}} \Phi_{2}(\mathbf{r}, t)+\Sigma_{s_{12}} \Phi_{1}(\mathbf{r}, t), \\
& \frac{\partial}{\partial t} C(\mathbf{r}, t)=\beta\left(\Sigma_{f_{1}} \Phi_{1}(\mathbf{r}, t)+v \Sigma_{f_{2}} \Phi_{2}(\mathbf{r}, t)\right)-\lambda C(\mathbf{r}, t) ;
\end{aligned}
$$

where $\Phi_{1}(\mathbf{r}, t)$ and $\Phi_{2}(\mathbf{r}, t)$ are fast and thermal neutron flux, $C(\mathbf{r}, t)$ is average one group of the precursor concentration of delayed neutrons, $D_{1}$ and $D_{2}$ are fast and thermal diffusion coefficients, $\Sigma_{a_{1}}$ and $\Sigma_{a_{2}}$ are fast and thermal absorption cross-sections, $\Sigma_{f_{1}}$ and $\Sigma_{f_{2}}$ are fast and thermal fission cross-sections, $\Sigma_{s_{12}}$ is the scattering crosssection from fast-to-thermal neutron, $v$ is the neutron fission, $v_{1}$ and $v_{2}$ are fast and thermal neutron speed, $\lambda$ is decay constant of average one group of delayed neutrons, and $\beta$ is the fraction of delayed neutrons.

The neutron flux and the precursor concentration of delayed neutrons can be written as separable functions of space and time as 


\section{International Journal of Science and Research (IJSR) \\ ISSN (Online): 2319-7064 \\ Index Copernicus Value (2013): 6.14 | Impact Factor (2014): 5.611}

$$
\begin{aligned}
& \Phi_{1}(\mathbf{r}, t)=v_{1} n_{1}(t) \hat{\Psi}(\mathbf{r}) \\
& \Phi_{2}(\mathbf{r}, t)=v_{2} n_{2}(t) \hat{\Psi}(\mathbf{r}) ; C(\mathbf{r}, t)=c(t) \hat{\Psi}(\mathbf{r})
\end{aligned}
$$

where $n_{1}(t)$ and $n_{2}(t)$ are the fast and thermal neutron density, $c(t)$ is the precursor concentration density of delayed neutrons and $\hat{\Psi}(\mathbf{r})$ is the fundamental function which can be determined from the following:

$$
\nabla^{2} \hat{\Psi}(\mathbf{r})+B^{2} \hat{\Psi}(\mathbf{r})=0
$$

where $B^{2}$ is the material buckling. Substituting (19) and (20) into (18) yields

$$
\begin{aligned}
& \frac{d n_{1}(t)}{d t}=-D_{1} B^{2} v_{1} n_{1}(t)-\left(\Sigma_{a_{1}}+\Sigma_{s_{12}}\right) v_{1} n_{1}(t) \\
& +v \Sigma_{f_{1}}(1-\beta) v_{1} n_{1}(t)+v \Sigma_{f_{2}}(1-\beta) v_{2} n_{2}(t)+\lambda c(t), \\
& \frac{d n_{2}(t)}{d t}=-D_{1} B^{2} v_{2} n_{2}(t)-\Sigma_{a_{2}} v_{2} n_{2}(t)+\Sigma_{s_{12}} v_{1} n_{1}(t) \\
& \frac{d c(t)}{d t}=\beta\left(v \Sigma_{f_{1}} v_{1} n_{1}(t)+v \Sigma_{f_{2}} v_{2} n_{2}(t)\right)-\lambda c(t) .
\end{aligned}
$$

Let us consider that $l_{1}=1 / v_{1} v \sum_{f_{1}}$ and $l_{2}=1 / v_{2} v \sum_{f_{2}}$ are fast and thermal generation time of neutrons, $L_{1}^{2}=D_{1} / \Sigma_{a_{1}}$ and $L_{2}^{2}=D_{2} / \Sigma_{a_{2}}$ are fast and thermal diffusion length, $k_{1}=v \Sigma_{f_{1}} / \Sigma_{a_{1}}\left[1+L_{1}^{2} B^{2}\right]$ and $k_{2}=v \Sigma_{f_{2}} / \Sigma_{a_{2}}\left[1+L_{2}^{2} B^{2}\right]$ are fast and thermal multiplication factor, $\rho_{1}=\left(k_{1}-1\right) / k_{1}$ and $\rho_{2}=\left(k_{2}-1\right) / k_{2}$ are fast and thermal reactivities, $\kappa=v_{1} \Sigma_{s_{12}}, \mu_{1}=\beta / l_{1}$ and $\mu_{2}=\beta / l_{2}$.

Equations (21) are rewritten in the following form

$$
\begin{aligned}
& \frac{d n_{1}(t)}{d t}=\left(\frac{\rho_{1}}{l_{1}}-\mu_{1}-k\right) n_{1}(t) \\
& +\left(\frac{1}{l_{2}}-\mu_{2}\right) n_{2}(t)+\lambda c(t), \\
& \frac{d n_{2}(t)}{d t}=\kappa n_{1}(t)+\left(\frac{\rho_{2}-1}{l_{2}}\right) n_{2}(t) \\
& \frac{d c(t)}{d t}=\mu_{1} n_{1}(t)+\mu_{2} n_{2}(t)-\lambda c(t), \\
& \frac{d}{d t} \Psi(t)=\mathbf{A} \Psi(t)
\end{aligned}
$$

where

$$
\Psi(t)=\left[\begin{array}{c}
n_{1}(t) \\
n_{2}(t) \\
c(t)
\end{array}\right] ; \quad \mathbf{A}=\left[\begin{array}{ccc}
-\alpha & \zeta & \lambda \\
\kappa & -\eta & 0 \\
\mu_{1} & \mu_{2} & -\lambda
\end{array}\right], \begin{gathered}
\alpha=\mu_{1}+\kappa-\rho_{1} / l_{1} \\
\zeta=1 / l_{2}-\mu_{2} \\
\eta=\left(1-\rho_{2}\right) / l_{2}
\end{gathered}
$$

Using the exponential integrator, we can determine the general solution of (23) as follows:

$$
\Psi(t)=B \exp \left(\int_{t_{0}}^{t} \mathbf{A} d t\right)
$$

where $\mathrm{B}$ is the integral constant.

This solution includes the exponential function of the matrix A which can be calculated using generalization of the analytical exponential as

$$
\Psi(t)=\sum_{k=1}^{3} B_{k} \exp \left(\omega_{k} t\right) \mathbf{U}_{k}
$$

where $B_{k}$ are the constants, $\omega_{k}$ are the eigenvalues of the matrix $\mathbf{A}$ and $\mathbf{U}_{k}$ are the corresponding eigenvectors of the matrix A.

Using the initial condition $\Psi(0)=\Psi_{0}$ to determine the constants $B_{k}=\mathbf{V}_{k}^{T} \Psi_{0}$, (26) becomes as follows

$$
\Psi(t)=\sum_{k=1}^{3} \exp \left(\omega_{k} t\right) \mathbf{U}_{k} \mathbf{V}_{k}^{T} \Psi_{0}
$$

where, $\mathbf{V}_{k}$ are the eigenvectors of matrix $\mathbf{A}^{T}$ which corresponding to eigenvalues $\omega_{k}$ and satisfies the normalization condition $\mathbf{U}_{k}^{T} \mathbf{V}_{k}=1$.

Equation (27) gives good results only for a constant matrix A. But for variable matrix $\mathbf{A}$, the solution of the equation (23) can be takes the form

$$
\Psi\left(t_{m+1}\right)=\sum_{k=1}^{3} \exp \left(h \omega_{k}\right) \mathbf{U}_{k} \mathbf{V}_{k}^{T} \Psi\left(t_{m}\right)
$$

where, the values of the eigenvalues $\omega_{k}$ and the corresponding eigenvectors $\mathbf{U}_{k}$ and $\mathbf{V}_{k}$ are calculated at time $t_{m}+(h / 2)$ and $h=t_{m+1}-t_{m}$ is the time step interval $\left[t_{m}, t_{m+1}\right]$.

Equations (27) and (28) represent the solutions of the two energy groups of point kinetics equation with average one group of delayed neutrons. These solutions represent a generalization of the analytical exponential model for step and variable coefficient matrix A, respectively.

The eigenvalues of the matrix $\mathbf{A}$ are determined from the following algebraic equation: 
$\omega^{3}+\omega^{2}(\alpha+\eta+\lambda)+\omega\left(\alpha \eta+\alpha \lambda+\eta \lambda-\kappa \zeta-\lambda \mu_{1}\right)$

$+\alpha \eta \lambda-\kappa \zeta \lambda-\kappa \lambda \mu_{2}-\eta \lambda \mu_{1}=0$

This equation is easy to find the corresponding solutions. The eigenvectors of the matrix A are determined analytically as follows:

$$
\mathbf{U}_{k}=\left[\begin{array}{c}
1 \\
\frac{\kappa}{\omega_{k}+\eta} \\
\frac{\mu_{1}\left(\omega_{k}+\eta\right)+\kappa \mu_{2}}{\left(\omega_{k}+\lambda\right)\left(\omega_{k}+\eta\right)}
\end{array}\right]
$$

The eigenvalues of matrix $\mathbf{A}^{T}$ easily be calculated. It follows

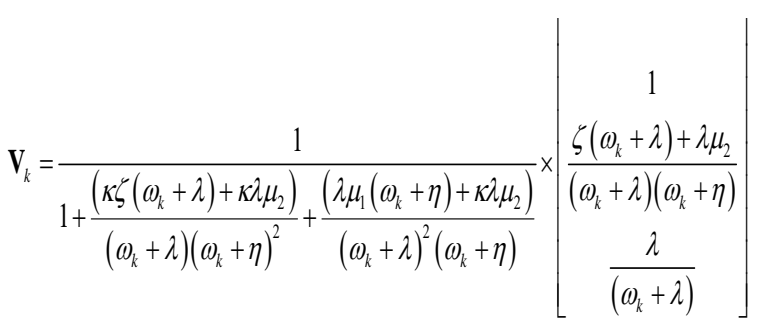

\section{Conclusions}

The numerical calculations based on the finite difference method to form nuclear reactor geometry standards. We have put in $\mathrm{AD}$ method to handle the discontinuity or crack boundary geometry in combination with the basic matrix method and analytical methods for the numerical solution to the diffusion equation of the second group of energy and energy group for delayed neutrons. The solution method is extended to all the meshing of the reactor, including the meshing of a nuclear reactor with standard 3-dimensional geometry (benmark reactor). They are compared with the traditional method and it can be concluded that the inclusion of $\mathrm{AD}$ method for higher accuracy than other methods, because only the domain algorithm, points bizarre overcome by religion average levels. High precision with domain 2 and 3-dimensional geometry, evenly distributed by pretreatment of the initial data by averaging integral sense. At regular points similar results, at the singularity (including on the boundary) than the result, particularly good results in accordance with standard reactor and heterogeneous reactor.

\section{Acknowledgments}

I would like to gratefully and sincerely thank to Prof. P.V. Hap for his guidance and for his suggestion for many valuable comments to this article. This research is supported by the Nafosted No. $103.03-2012.2$.

\section{References}

[1] W. M. Stacey, Nuclear Reactor Physics, John Wiley \& Sons, New York, NY, USA, 2001.
[2] G. Arfken and H. Weber, Mathematical Methods for Physicists (5th edition, Academic Press, San Diego), 2000.

[3] K.S. Smith, An analytic nodal method for solving the two-group, multidimensional, static and transient neutron diffusion equations, M. Sc. Thesis, Nuclear Engineering, Massachusetts Institute of Technology, 1979.

[4] R.N. Blomquist and E.M. Gelbard, Alternative Implementations of the Monte Carlo Power Method, Nucl. Sci. Eng., 141, 85 (2003).

[5] D.L. Hetrick, Dynamics of Nuclear Reactors, American Nuclear Society, La Grange Park, 1993.

[6] E.G. Whitesides, Difficulty in Computing the k-Effective of the World, Trans. Am. Nucl. Soc., 14, 680 (1971).

[7] N.Z. Cho, et al., Refinement of the 2-D/1-D Fusion Method for 3-D Whole-Core Transport Calculation, Trans. Am. Nucl. Soc., 87, 417 (2002).

[8] A. Quarteroni, R. Sacco and F. Saleri, Numerical Mathematics, Springer-Verlag, New York, Inc. USA, 2000.

[9] S. Glasstone and A. Sesonske, Nuclear Reactor Engineering, Chapman \& Hall, 1994.

[10]N.Z. Cho, Fundamentals and Recent Developments of Reactor Physics Methods, Nuclear Engineering and Technology, 37, 25 (2005).

[11] Joseph J. Golecki, Direct displacement method in crack theory (numerical resolution), Meccanica, Volume 42, Issue 6, pp 555-566. 2007.

[12]Hoang Anh Tuan, Phan Huy Thien, Phan Van Hap. Advanced precision approximate solution of equations by means of continuous parameters. Proceedings of International Conference on Physics of nuclear reactor 19 th. Budapest, Hungary, 193 - 201, 1990.

[13] Phan Van Hap, Phan Huy Thien. The AD Method for the approximate solution of the Wigner Equations. Demonstration Mathematica Vol XXVII, No 2. pp283286, 1994 